\title{
Pregnancy outcome in women with subchorionic haemorrhage detected in first and second trimester of pregnancy
}

\begin{tabular}{|c|c|c|}
\hline Parez Retha* & Samira S. Shekh Mohammed ** & Parween Omer Hassen $* * *$ \\
\hline & Abstract & \\
\hline
\end{tabular}

Background and objective: Subchorionic hemorrhage is the most common sonographic abnormalities and the most common cause of first trimester miscarriage and mainly associated with vaginal bleeding. The present study was conducted with an aim to find out the effect of subchorionic hemorrhage in first and second trimester of pregnancy outcome.

Methods: A case cohort prospective study was designed to investigate 100 patients having a subchorionic hematoma in the presence of singleton live embryo or fetus. The study assessed the association of the volume size of subchorionic hematoma, maternal age and gestational age at time of the diagnosis with adverse pregnancy outcomes.

Results: The incidence of miscarriage and preterm delivery was $35 \%$ and $21 \%$ respectively. The mean $( \pm S D)$ age of those who aborted was $29.97 \pm 7.0$ years, slightly higher than the mean age of those who ended with preterm delivery (27.7 \pm 5.87 years) and those continued with their pregnancies to viability $(28.3 \pm 6.2$ years). There was a statistically significant association between previous histories of preterm delivery and pregnancy outcome $(P=0.015)$. Miscarriage was significantly high among women with gestational age $<12$ weeks, while term pregnancy was significantly high among women with gestational age $>13$ weeks $(P<0.001)$. The estimated volume of the hematoma did not correlate with the outcome of the pregnancy, perhaps it is the presence or absence of a hematoma as a marker of the integrity of placentation and not its size.

Conclusion: Subchorionic hematoma in first and second trimester of pregnancy associated with adverse pregnancy outcome (spontaneous miscarriage and preterm delivery.

Keywords: Subchorionic hemorrhage, Ultrasonography, Pregnancy outcome.

\section{Introduction}

Subchorionic hematoma is the pooling of blood between the chorion and membrane surrounding the embryo, and the uterine wall. With an incidence of $3.1 \%$ of all pregnancies, ${ }^{1,2}$ it is the most common sonographic abnormalities and the most common cause of first trimester miscarriage. The reported incidence of intrauterine hematoma has a wide range, between $0.5 \%$ and $22 \%$, mainly associated with vaginal bleeding. The discrepancy in these rates might be related to different patient populations and or study design.,4 Ultrasound is the imaging modality of choice for subchorionic hematoma, because it can be performed rapidly at the patient bed side and it has no risks to the pregnancy. It appears as an anechoic area that has a falciform shape, and it is usually observed behind or below the gestational sac, separating the chorion from the inner wall of the uterus, ${ }^{3-5}$ but the sensitivity of sonography is low, varies between $2-20 \%$ as blood may pass vaginally and not collected in sub chorionic space. $^{2,4,6}$ Subchorionic hematoma often regresses specially if small or moderate in size; large hematoma which strips at least $30-40 \%$ of placenta away from endometerium may enlarge further compressing the gestational sac with

* Department of Gynecology and Obstetrics, College of medicine, Hawler Medical University, Erbil, Iraq.

* Department of Gynecology and Obstetrics, Erbil Maternity Hospital, Erbil, Iraq.

* Department of Ultrasonogaphy, Erbil Maternity Hospital, Erbil, Iraq. 
Consequent spontaneous miscarriage..$^{2,4,7}$ The symptoms and signs are so variable that the patient may have bleeding of varying quantities ranging from light spotting to heavy flow with clot. The bleeding can be painless or there may be mild abdominal cramping.$^{6-9}$ Previously published studies show conflicting results. Some investigators have found associations between subchorionic hemorrhage and multiple adverse pregnancy outcomes, including preterm labor, spontaneous miscarriage, placental abruption, intrauterine growth restriction, intrauterine fetal demise. ${ }^{1,2,10}$ Usually, we cannot identify any cause for a subchorionic clot. Pre-existing medical conditions, autoimmune diseases, and immunological factors have been associated with intrauterine hematoma, but the etiology of this condition is still unknown. ${ }^{11,12}$ To authors' knowledge, no research has been conducted in Erbil Maternity Teaching Hospital to determine the effect subchorionic hematoma on pregnancy outcome. Therefore, this study was conducted with an aim to find out the effect of subchorionic hemorrhage in first and second trimester of pregnancy outcome.

\section{Methods}

This case cohort designed prospective study was carried out between May, 2013 andMay2014. One hundred women were included in the current study. Patients who were referred to Maternity Teaching Hospital in Erbil city, Kurdistan Region, Iraq, for ultrasound examination purpose because of vaginal bleeding in the first and second trimester of pregnancy and those who were found to have a subchorionic hematoma in the presence of singleton live embryo or fetus were included in this study after giving their consent to participate in the trial. The base line data were recorded on a questionnaire by direct interview with the patients. Patients with a non-viable pregnancy, fetal abnormality, multiple pregnancy and patients with medical diseases were excluded from the study.
Gestational age was calculated on the basis of the last menstrual period and was corrected with the crown-rump length measurements. The volumes of the hematomas were estimated by measuring the maximum transverse, antero-posterior, and longitudinal diameters, multiplying these values by a constant of 0.523 as was suggested by Nagy et al. ${ }^{10}$ The correction factor of 0.52 is used to correct for the crescent shape of the hematoma. All were followed with repeated ultrasound at seven days interval until miscarriage occurred or bleeding ceased, and the subchorionic hematoma disappeared, and then followed up by phone until delivery. The main outcome measures that included size of haematoma, gestational age and maternal age were compared for adverse pregnancy outcome (preterm labor, miscarriage, fetal birth weight) as well as term birth. For the analysis, the gestational age of 12 weeks, and the size of $10 \mathrm{~cm}^{3}$ of hematoma were taken as cut-off value. The study protocol was approved by the Scientific and Ethical Committee of College of Medicine of Hawler Medical University and an informed consent was obtained from all the patients. Statistical package for the social science (version 18) was used for data entry and analysis. Two approaches used; descriptive for determining of frequencies and mean standard deviations, while analytic approach was used through Chi-square test for categorical variables and independent $t$ test for determination of mean of continuous variables. $P$ value of $\leq 0.05$ regarded as statistically significant.

\section{Results}

In the current study 100 women; their mean $( \pm S D)$ of age was $28.75 \pm 6.4$ years with age ranged from 18 to 42 years, showed ultrasonographic evidence of a live fetus and subchorionic hematoma. The pregnancy of $35 \%$ of the study participants ended by miscarriage, while the pregnancy of $21 \%$ and $44 \%$ ended by preterm and term delivery, respectively. The gestational age of $70 \%$ of the study participants was 
less than 12 weeks. The normal range of term fetal weight was 2.5-3.5 kg. Among 65 patients who reached term and preterm pregnancy, the mean fetal weight was $2.8 \pm 0.82 \mathrm{~kg}$, while the mean fetal weight of $44 \%$ of patients with term pregnancy was $3.3 \pm 0.5 \mathrm{~kg}$, which was higher than the mean ( \pm SD) fetal weight of preterm delivery $(1.7 \pm 0.2), \quad P<0.001$. The size of hematoma of $59 \%$ of patients was less than $5 \mathrm{~cm}^{3}$, and $23 \%$ of patient had a hematoma more than $10 \mathrm{~cm}^{3}$ in size (Table 1). This study revealed a statistically significant association between previous histories of preterm delivery and pregnancy outcome, in which more than half percent of those who had previous history of preterm pregnancy ended with preterm delivery $(P=0.015)$, while there was no significant association between previous miscarriage and pregnancy outcome $(P=0.337)$ as shown in Table 2.

Table 1: Certain characteristics of the study sample.

\begin{tabular}{lll}
\hline Variables & $\mathbf{N}$ & $\mathbf{( \% )}$ \\
\hline Age & 3 & $(3.00)$ \\
$\leq 18$ & 26 & $(26.0)$ \\
$18-24$ & 27 & $(27.0)$ \\
$25-29$ & 22 & $(22.0)$ \\
$30-34$ & 13 & $(13.0)$ \\
$35-39$ & 9 & $(9.00)$ \\
$\geq 40$ & 100 & $(100)$ \\
Total & & \\
Gestational age & 70 & $(70.0)$ \\
$\leq 12$ & 30 & $(30.0)$ \\
$>12$ & 100 & $(100)$ \\
Total & & \\
Fetal weight $(\mathbf{N}=65)^{*}$ & 20 & $(30.8)$ \\
$<2$ & 22 & $(33.8)$ \\
$2-3$ & 23 & $(35.4)$ \\
$>3$ & 65 & $(100)$ \\
Total & & \\
Size of hematoma $\mathbf{c m}^{3}$ & 59 & $(59.0)$ \\
$<5$ & 18 & $(18.0)$ \\
$5-9$ & 23 & $(23.0)$ \\
$\geq 10$ & 100 & $(100)$ \\
Total & &
\end{tabular}

${ }^{*}$ Miscarriage excluded

Table 2: Association between certain variables with pregnancy outcome.

\begin{tabular}{|c|c|c|c|c|c|c|c|c|c|}
\hline \multirow{3}{*}{ Variables } & \multicolumn{6}{|c|}{ Pregnancy outcomes } & \multirow{2}{*}{\multicolumn{2}{|c|}{$\begin{array}{l}\text { Total } \\
(\mathrm{N}=100)\end{array}$}} & \multirow{3}{*}{$\mathbf{P}$} \\
\hline & \multicolumn{2}{|c|}{ Miscarriage } & \multicolumn{2}{|c|}{ Preterm delivery } & \multicolumn{2}{|c|}{ Term delivery } & & & \\
\hline & No. & $\%$ & No. & $\%$ & No. & $\%$ & No. & $\%$ & \\
\hline \multicolumn{10}{|c|}{ Previous miscarriage } \\
\hline No & 26 & 31.7 & 18 & 22.0 & 38 & 46.3 & 82 & 100 & \multirow[b]{2}{*}{0.337} \\
\hline Yes & 9 & 50.0 & 3 & 16.7 & 6 & 33.3 & 18 & 100 & \\
\hline \multicolumn{10}{|c|}{ Previous preterm } \\
\hline No & 33 & 37.1 & 15 & 16.9 & 41 & 46.1 & 89 & 100 & \multirow{2}{*}{0.015} \\
\hline Yes & 2 & 18.2 & 6 & 54.5 & 3 & 27.3 & 11 & 100 & \\
\hline
\end{tabular}


Miscarriage was significantly high among women with gestational age $\leq 12$ and term pregnancy was significantly high among women with gestational age $>12$ weeks $(P=0.001)$ and shown in Table 3. Among three groups of patients with miscarriages, preterm and term deliveries, that had subchorionic hematoma in early pregnancy, the mean age of those who aborted was (29.97 \pm 7.0 years), slightly higher than the mean age of those who ended with preterm delivery $(27.7 \pm 5.87$ years) and those continued with their pregnancies to viability $(28.3 \pm$ 6.2 years). Regarding age of women and pregnancy outcome, miscarriage rate increased with advancing age, but statistically was not significant $(P=0.517)$ as shown in Table 4. A statistically non- significant association was found between size of haematoma and the outcome of pregnancy $(P=0.654)$ as shown in Table 5 . The current study revealed a statistically significant association between gestational age and size of hematoma $(P<0.001)$ as shown in Table 6 .

Table 3: Gestational age at diagnosis of hematoma and pregnancy outcome among studied sample.

\begin{tabular}{llllllllll}
\hline & \multicolumn{4}{l}{ Pregnancy outcomes } & \multicolumn{3}{l}{ Total } \\
Gestational age & \multicolumn{2}{l}{ Miscarriage } & \multicolumn{2}{c}{ Preterm delivery } & \multicolumn{2}{c}{ Term delivery } & \multicolumn{2}{c}{ (N=100) } & P \\
& No. & $\%$ & No. & $\%$ & No. & $\%$ & No. & $\%$ & \\
\hline$\leq 12$ & 32 & 45.7 & 10 & 14.3 & 28 & 40 & 70 & 100 & 0.001 \\
$>12$ & 3 & 10.0 & 11 & 36.7 & 16 & 53.3 & 30 & 100 & \\
\hline
\end{tabular}

Table 4: Association between maternal age and pregnancy outcome among studied sample.

\begin{tabular}{lllllllllll}
\hline & \multicolumn{4}{l}{ Pregnancy outcomes } & \multicolumn{3}{c}{ Total } \\
Maternal Age in Years & \multicolumn{2}{l}{ Miscarriage } & \multicolumn{2}{l}{ Preterm delivery } & \multicolumn{2}{c}{ Term delivery } & \multicolumn{2}{c}{ (N=100) } & P \\
& No. & $\%$ & No. & $\%$ & No. & $\%$ & No. & $\%$ & \\
\hline $18-23$ & 7 & 29.2 & 7 & 29.2 & 10 & 41.7 & 24 & 100 & \\
$24-29$ & 11 & 34.4 & 4 & 12.5 & 17 & 53.1 & 32 & 100 & 0.517 \\
$\geq 30$ & 17 & 38.6 & 10 & 22.7 & 17 & 38.6 & 44 & 100 & \\
\hline
\end{tabular}

Table 5: Association between size of haematoma and pregnancy outcome.

\begin{tabular}{|c|c|c|c|c|c|c|c|c|c|}
\hline \multirow{3}{*}{$\begin{array}{l}\text { Size of hematoma } \\
\left(\mathrm{cm}^{3}\right)\end{array}$} & \multicolumn{6}{|c|}{ Pregnancy outcomes } & \multirow{2}{*}{\multicolumn{2}{|c|}{$\begin{array}{l}\text { Total } \\
(\mathrm{N}=100)\end{array}$}} & \multirow{3}{*}{$\mathbf{P}$} \\
\hline & \multicolumn{2}{|c|}{ Miscarriage } & \multicolumn{2}{|c|}{ Preterm delivery } & \multicolumn{2}{|c|}{ Term delivery } & & & \\
\hline & No. & $\%$ & No. & $\%$ & No. & $\%$ & No. & $\%$ & \\
\hline$<5$ & 24 & 40.7 & 11 & 18.6 & 24 & 40.7 & 59 & 100 & \\
\hline $5-9$ & 4 & 22.2 & 5 & 27.8 & 9 & 50.0 & 18 & 100 & 0.654 \\
\hline$\geq 10$ & 7 & 30.4 & 5 & 21.7 & 11 & 47.8 & 23 & 100 & \\
\hline
\end{tabular}

Table 6: Association between gestational ages with size of hematoma.

\begin{tabular}{|c|c|c|c|c|c|c|c|c|}
\hline \multirow{3}{*}{ Gestational age } & \multicolumn{6}{|c|}{ Size of hematoma $\mathrm{cm}^{3}$} & \multirow{2}{*}{\multicolumn{2}{|c|}{ Total }} \\
\hline & \multicolumn{2}{|c|}{$<5$} & \multicolumn{2}{|l|}{$5-9$} & \multicolumn{2}{|c|}{$\geq 10$} & & \\
\hline & No & $\%$ & No & $\%$ & No & $\%$ & No & $\%$ \\
\hline$\leq 12$ & 50 & 71.4 & 11 & 15.7 & 9 & 12.9 & 70 & 100 \\
\hline$\geq 13$ & 9 & 30.0 & 7 & 23.3 & 14 & 46.7 & 30 & 100 \\
\hline Total & 59 & 59.0 & 18 & 18.0 & 23 & 23.0 & 100 & 100 \\
\hline$P$ value & $<0$ & & & & & & & \\
\hline
\end{tabular}




\section{Discussion}

During the period of study, 100 women showed ultrasonographic evidence of live fetus and subchorionic haematoma. Patients in current study are not representative of all patients with subchorionic hematoma in Erbil city or in Maternity Teaching Hospital because the unit accepts emergency cases only. Therefore, we could not calculate the incidence of hematoma in our population. The percentage of miscarriage in our study was $35 \%$ and the percentage of preterm and term delivery was $21 \%$ and $44 \%$, respectively. Preterm delivery ( $<37$ weeks) was the most frequently investigated outcome in patients with subchorionic and retro placental hematomas. Our report of a $21 \%$ rate of preterm delivery is consistent with the $19 \%$ reported by Loi and Tan. ${ }^{7}$ It has been suggested that a localized accumulation of blood causes mechanical uterine irritation and therefore stimulates contractions. According to our references none of the prior studies focused on known risk factors like previous preterm delivery for adverse pregnancy outcome, but our study revealed statistical association between previous history of preterm delivery and current preterm delivery $(P=0.015)$. Whereas, there was no correlation between previous history of miscarriage and current miscarriage ( $P$ $=0.337$ ). Gestational age at diagnosis was another variable. This study revealed that miscarriage was significantly high among women with gestational age less than 12 weeks while term pregnancy was significantly high among women with gestational age more than 12 weeks, $P<0.001$. Dealing with subchorionic hematoma and maternal age, Bennett et al 2 revealed that the spontaneous abortion rate was approximately twice as high for women aged 35 years or older in comparison to younger women $(13.8 \%$ and $7.3 \%$, respectively). This study revealed that spontaneous miscarriage rate slightly increased with advancing age (>30y) as for younger age (38.6\% and
$29.7 \%$, respectively), but this did not reach statistical significance $(P=0.517)$, probably because of the small sample size of the study. The results from available studies on hematoma volume and pregnancy outcome are again controversial. Many observational reports revealed a significant correlation between "large" hematomas and adverse outcome of pregnancy, ${ }^{11,12}$ while others failed to demonstrate this association. ${ }^{9,10}$ In our current study the size of $10 \mathrm{~cm}^{3}$ of hematoma was taken as the cut-off value a statistically non-significant association was found between size of hematoma and the outcome of pregnancy $(P=0.654)$. Our results was different from those of Bennett et $\mathrm{al}^{2}$ who found that large volumes increased 2.4-fold the risk of spontaneous miscarriage. These different results might be the consequence of our limited sample size or might be explained by that the majority of our cases showed small hematomas (volumes $<5 \mathrm{ml}$ were observed in $59 \%$ of the cases). The current study revealed a statistically significant association between gestational age and size of hematoma $(P<0.001)$. This result is similar to research that done by Kurjak et al. ${ }^{5}$ Finally about the risks of other late complications of pregnancy such as stillbirth, abruption placenta, intrauterine growth restriction, fetal distress, and preeclampsia, when intrauterine hematoma is diagnosed many of these clinical conditions are a consequence of impaired placentation, and it might be postulated that the presence of a subchorionic hematoma in early stages affects the normal process of trophoblast invasion this need further researches in the future.

\section{Conclusion}

Our study concluded that the presence of an intrauterine hematoma in first and second trimester of pregnancy is associated with adverse pregnancy outcome (spontaneous miscarriage and preterm delivery). Although there are no specific management interventions to prevent the adverse outcomes, women 
with subchorionic hemorrhage should receive more precise counseling as to the risks to their pregnancy may increase risk for adverse pregnancy outcome.

\section{Conflicts of interest}

The authors report no conflicts of interest.

\section{References}

1. Abu-Yousef MM, Bleider JJ, Williamson RA, Weiner CP. Subchorionic hemorrhage: Sonographic diagnosis and clinical significance. Am J Roentgenol 1987;149:737-40.

2. Bennett GL, Bromley B, Lieberman E, Benacerraf BR. Subchorionichemorrhage in first-trimester pregnancies: prediction of pregnancyoutcome with sonography. Radiology 1996; 200:803-6.

3. Kyser KL. Meta-analysis of subchorionic haemorrhage and adverse pregnancy outcome. ProcObstetGynecol2012; 2(4)

4. Mantoni M. Ultrasound signs in threatened abortion and their prognostic significance. ObstetGynecol 1985; 65:471-5.

5. Kurjak A, Schulman H, Zudenigo D, Kupesic S, Kos M, Goldenberg M. Subchorionic hematomas in early pregnancy: clinical outcome and blood flow patterns. Maternal Fetal Medicine 1996; 5: 41-4.

6. Baxi LV, Pearlstone MM. Subchorionic hematomas and the presence of autoantibodies. Am JObstetGynecol 1991; 165:1423-4.

7. Loi K, Tan K T. Massive pre-placental and subchorionichaematoma. Singapore Med J 2006; 47(12) :1085.

8. LieteJ, Ross P, Rossi AC, Philippe J. Prognosis of very large first trimester heamatoma. Ultrasound Med 2006; 25:1441-5.

9. Pedersen JG, Mantoni M. Large intrauterine hematoma in threatened miscarriage. Frequency and clinical consequences. $\mathrm{Br} \mathrm{J}$ Obstetric Gynaecol1990; 97:75-7.

10. Nagy S, Bush M, Stone J, Lipinski RH, Gardo S. Clinical significance of sub chorionic and retro placental hematomas detected in the first trimester of pregnancy. Obstetric Gynecol 2003; 102(1):94-100.

11. Johns J, Hyett J, Jauniaux E. Obstetric outcome after threatened miscarriage with and without a hematoma on ultrasound. Obstetric Gynecol 2003; 102(3):483-7.

12. Saurbrei EE, Pham DH. Placental abruption and sub chorionic hemorrhage in the first half of pregnancy: US appearance and clinical outcome. Radiology 1986; 160:109-12. 